\title{
Remote Sensing Payload Development for High Altitude Balloons
}

\author{
Zsófia Bodó \& Bence Dávid Góczán \\ Simonyi Karoly College for Advanced Studies \\ Budapest, Hungary \\ bodo.zsofia@simonyi.bme.hu,goczan.bence@simonyi.bme.hu
}

\begin{abstract}
Remote sensing provides fundamental data about landmark characteristics. Up to date remote sensing information is vital in almost every industrial and agricultural sector. Providing such data and processing services are interesting fields for both traditional space agencies and smaller new-space companies. However there is one method of data acquisition that is not yet widely utilized. With a stratospheric balloon large areas could be covered and balloons can be launched in a frequent manner. The UPRA Project (Universal Platform for Robotics and Aerospace) is a student project with an aim to develop a reliable, widely configurable high altitude balloon platform for university research groups.
\end{abstract}

The aim of the project is to build a proof-of-concept multispectral remote sensing hardware and using open source and self-developed software to analyze data provided by the payload to demonstrate that high quality and relevant remote sensing can be achieved for a comparably low price. The payload train consists of a parachute system, an avionics module, a backup GPS tracker, radar reflector, a flight termination unit, a control camera and the remote sensing module (UPRACAM), developed internally by the team. The avionics module contains the main flight computer, which provides two way radio communication, live telemetry and scientific data and also controls the payload.

A balloon-borne multispectral camera was developed that is capable to sense in visible and near infrared spectrum. The camera is made out of commercial off-the-shelf (COTS) components to keep the cost and development time low. The device has a twin-sensor configuration. The identical image sensors have a wide spectral response in the $420 \mathrm{~nm}-980 \mathrm{~nm}$ wavelength region which makes them ideal for this field of use. The camera lenses are equipped with short-pass filter for visible and high-pass filter for near infrared image capture.

Although the camera was developed for high altitude balloon, in the future it is possible to use it in small satellite missions since the design allows to easily integrate with CubeSat frames and various flight computers. In 2019 the project had four proof of concept flights with the developed remote sensing payload, collecting almost seven hours of flight data. This might be the first step to develop regular scientific remote sensing balloon missions in Hungary. During these flights the data collected might help authorities to organize protection or salvage during floods, after hails or storms. Also valuable information could be provided to the agriculture on vegetation covered areas and inland waters.

Keywords - high altitude balloon, remote sensing, earth observation, agriculture

\section{INTRODUCTION}

Nowadays remote sensing is a crucial part of our life. Different remote sensing platforms provide information about vegetation health, crop management, floods, inland-water, forest fires or even traffic. Data provided by remote sensing platforms is vital in almost every industrial and agricultural sector.

Remote sensing services became more and more popular in the new-space industry. Companies research new possibilities in both data gathering and processing services. Stratospheric balloons could be a reasonable candidate besides the already available remote sensing platforms by providing large, frequent coverage for a reasonable cost.

This paper introduces the possible use of small balloon platforms in remote sensing applications and the development of a small sized multispectral camera designed for high altitude balloons or picosatellite Earth observation missions. Successful test flights conducted with the proof of concept device indicates that the development is worth continue and could provide a competitive solution in the remote sensing domain.

\section{REMOTE SENSING PlATFORMS}

Gathering remote sensing data can be performed with different platforms. Using satellites are the most common method in Earth observation programmes. However, if the area of interest is smaller other different airborne platforms can be used, such as manned or unmanned aircrafts. Parameters - such as covered area, modularity, scalability or cost effectiveness can vary by each data gathering method. Effectiveness of a remote sensing programme can be enhanced by selecting the right platform according to these parameters. Beside the commonly used platforms there is a data gathering method that is relatively unresearched in commercial or regular remote sensing applications. High altitude balloons were one of the first remote sensing platforms but nowadays their significance is lower compared to satellites. However their application in smaller scale operations became a trending research topic among new-space companies.

As in Fig. 1 can be seen each platform performs well in at least one parameter, but these information should be handled together when a remote sensing mission is planned. Manned and unmanned aerial platforms provide high spatial resolution data due to their low altitude of operation, but they are not 
suitable to map large area. Due to the small coverage area and the need of frequent flights makes the cost per unit area of ground coverage relatively high with aerial vehicles. However these platforms usually used for one-time operations but in many areas continuous monitoring of Earth features is needed. Satellite platforms while providing lower resolution, they offer continuous or frequent coverage of an area of interest which makes the cost per unit area low. However the mission planning, development, validation, launch and control activities would increase the expenses for the overall mission which makes orbital platforms unaffordable for smaller research groups. High altitude balloons with a peak altitude of 40000 meters (operating in the stratosphere) can also cover significant area and with active altitude control it could perform the observation over the area of interest. Balloon platforms can be launched in a frequent manner to provide continuous coverage. Also there are research programmes working on continuous, actively controlled flock operation of balloons, to cover a designated area. On the basis of the cost parameter, the use of balloons should be considered. Expenses of one launch including payload mating, validation and launch activities can be solved from as little as 1000-1500 EUR for smaller sized payloads.
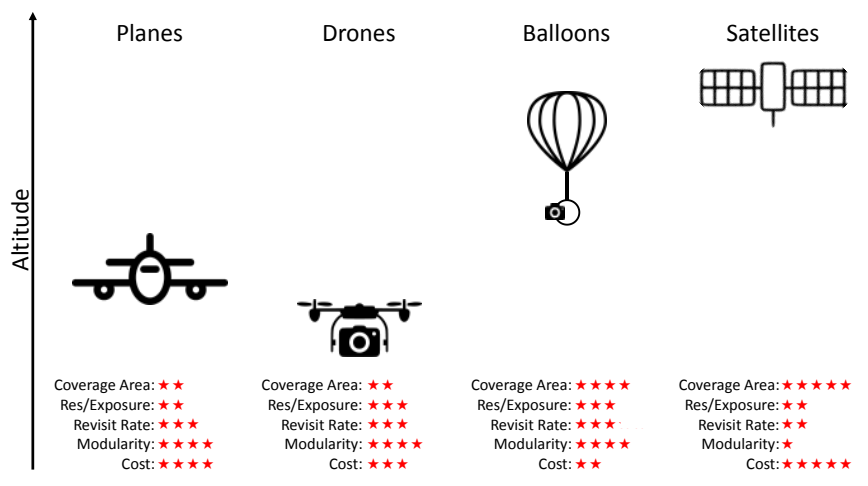

Fig. 1. Comparison of remote sensing platforms[1]

\section{UPRA BALLOON PLATFORM}

Universal Platform for Robotics and Aerospace (UPRA) Team of Simonyi Karoly College for Advanced Studies develops a configurable modular balloon platform. The platform is developed and manufactured by students and the goal of the project is to develop a launch platform for small science payloads up to 30 kilometers to the stratosphere. The UPRA Team is working on a launch service targeting university research groups to provide an affordable platform, launch, recovery and flight planning for their mission.

The platform has two way radio communication, live tracking, on-board data collection and data handling. The avionics module is also capable to control third party payloads and transmit science data during flight. The platform is equipped with redundant tracking system and an independent landing locator to ensure the successful recovery of the payload train.

To meet mission demands the platform is highly configurable and requires low maintenance which makes it suitable for frequent or regular stratospheric missions. It is planned to provide a standardized payload gondola to even lower the expenses and the preparation time, but if the mission requires custom made gondola also can be designed and mated with the payload train.

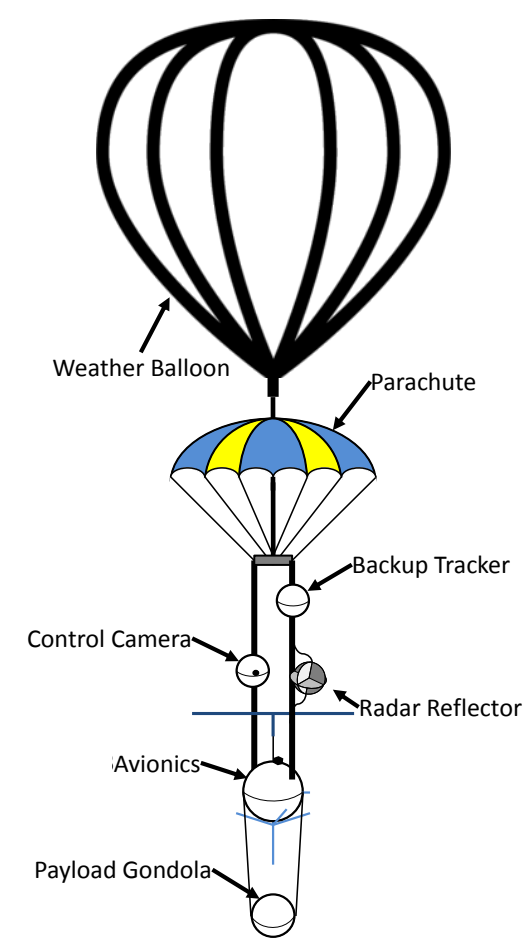

Fig. 2. UPRA payload train flight configuration[1]

Fig. 2 shows the payload train configuration with one payload gondola attached. Research is ongoing on the topic of multiple payload gondola configuration and its flight dynamics.

\section{REMOTE SENSING PAYLOAD}

As technological demonstration, a vegetation monitoring remote sensing payload (UPRACAM) is developed for UPRA balloon platform. This topic has been chosen due to its low development expense and the benefits of vegetation and open water surface observation on agriculture. Other methods could provide information on carbon-dioxide footprint, urbanization and traffic or temperature change of a region. These observations would need more delicate sensors and more expensive measurement hardware. Since the goal of this proof of concept mission is to pave the road for balloon borne remote sensing, a cost effective solution was chosen.

Vegetation and open water surfaces can be observed using a multispectral camera with a near-infrared (NIR) and a visible light (VIS) channel. Measurements could be fined if the VIS channel is divided to red (R), green $(G)$, blue (B) light.

Plants reflect mostly 500-560nm wavelength in the visible spectrum which makes them appear green. They also reflect $680-900 \mathrm{~nm}$ light which is in the near-infrared spectrum and cannot be seen by the human eye, although the amount of 
reflected NIR light carries important information about the health of the plant. Composite image information generated from NIR and VIS camera data can help monitoring and visualize vegetation health, plant composition and the proportion of vegetation covered areas. These data sets can help agricultural planning, crop and pesticide management. Beside information on plant life, data on open water surfaces is also very important in agrarian management to monitor inlandwater, canal structures and routes and to predict or calculate possible flood damage.

While plants reflects most of the near-infrared waves, open water surfaces reflects no or very little. With the same multispectral camera and image processing methods water covered areas can also be monitored. Using the composite images inland-water and flood-water area can be measured and used for crop management or property salvation.

\section{A. Hardware Implementation}

The sensor selected for the payload is the OV2640 CMOS image sensor which has large spectral sensitivity in both visible and infrared region (Fig. 3). The remote sensing multispectral camera is using two identical image sensor modules, one for visible light and one for near-infrared. A $800 \mathrm{~nm}$ shortpass filter is used on the visible module to cut the higher wavelengths, while a $800 \mathrm{~nm}$ longpass filter is applied on the NIR module to block the visible and UV, short wave light.

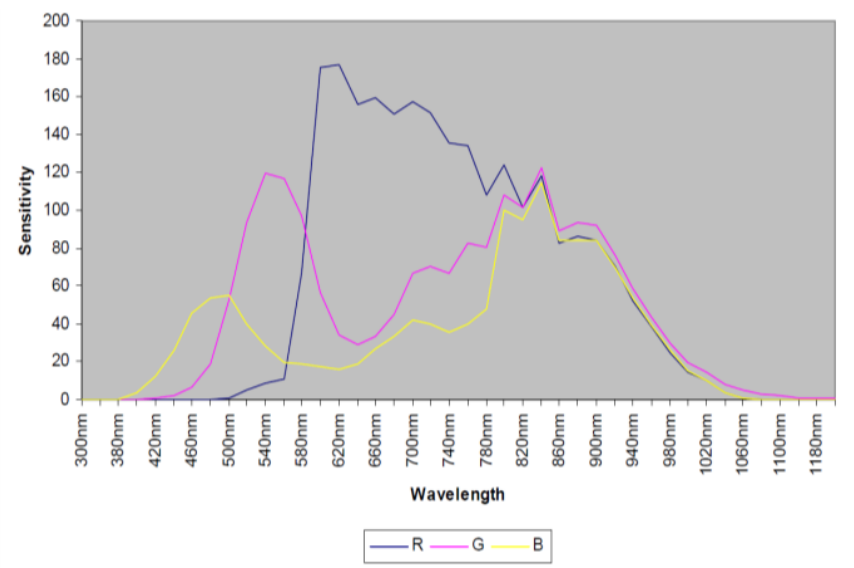

Fig. 3. OV2640 Spectrum Response [2]

The proof of concept model (Fig. 4) is based on two Arducam Mini 2Mpx modules with M12 (S-mount) lens mount, internal image buffer as temporary storage and OV2640 image sensor. The modules have separate image buffers which makes them suitable for simultaneous photo shooting. The NIR and VIS images are taken at the same time, stored temporarily on the sensor modules and stored on a non-volatile storage.

An Arduino compatible Microchip Atmega328p microcontroller is used to control and configure the sensors, handle the non-volatile storage and the file system. The control board is also provides power and data connection with the platform, the camera is developed to provide housekeeping data, low-resolution images for telecommunication and the possibility to set image properties by the ground control.
An SD Card is selected as non-volatile storage with FAT file system. The images saved on the SD Card with a running number and a channel indicator. Beside the pictures, the camera also stores control and configuration information on the storage. Prior flight it is possible to set the frame rate, resolution, mirroring, white balance and exposure by the configuration file stored on the SD Card. The running number is increased and stored after every frame taken. This prevents to overwrite older images during unplanned restart of the camera.

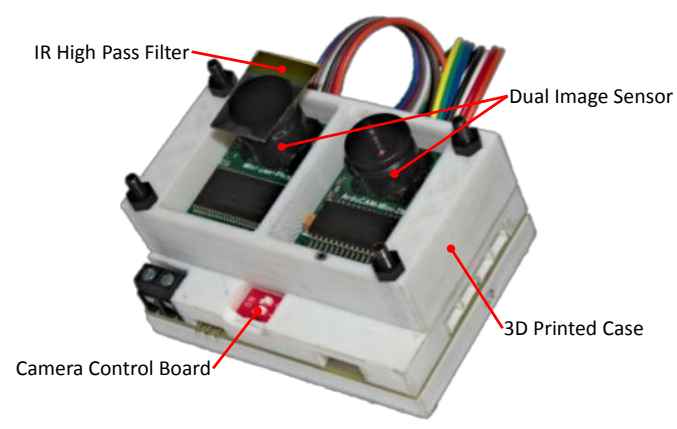

Fig. 4. UPRACAM Remote sensing camera prototype[1]

The enclosure of the camera is not larger than a typical action camera. It is $3 \mathrm{D}$ printed and designed to be able to be connected to passive and active stabilization systems. Currently during the test flights the camera was hanging at the end of the payload train without any stabilization or attitude control.

Since all used hardware elements are commercial-off-theshelf (COTS) components the validation of the payload is a crucial part of development and flight operations. To ensure maximum reliability the UPRACAM is tested after every small step of development or calibration. Environmental tests were also run in thermal chamber at $-50^{\circ} \mathrm{C}$ temperature. Prior flight, the a usual pre-flight smoke test is run on the camera module, and every integration step is followed a short validation process to check the proper operation of the camera.

\section{IMAGE PROCESSING}

Composite images are generated from VIS and NIR images using various image processing techniques. The processing can be performed by hand, but due to the large amount of data gathered during a flight it is worth to use automated processing algorithms. Two transformations were selected for further research in the project. NRG false coloring to highlight vegetation and Normalized Difference Vegetation Index (NDVI) visualized with color composite techniques.

The first step of both NRG and NDVI generation is to overlap the images. Since the NIR and VIS images are taken with different sensors the two pictures are never be fully identical. To perform an appropriate overlapping, calibration data should be provided. Lens calibration is a manual process when multiple images taken from multiple angles with a test pattern. 
The automated image processing algorithms are still under development in the time this paper is written. For the development, OpenCV libraries are used offering calibration recommendation, overlapping base functions and bit wise image processing methods. The image processing end product is planned to be a user friendly, configurable, modular software that will be able to handle different image transformations based on typical remote sensing application, and can be used on with various remote sensing payloads.

\section{A. NRG False Coloring}

NRG a false coloring method visualize the NIR light information and highlights the vegetation on composite images. The name 'NRG' refers to the color channels used in the image which are near-infrared $(\mathrm{N})$, red $(\mathrm{R})$ and green $(\mathrm{G})$.

The blue channel of the VIS image is completely discarded because shortwave light scatters in the atmosphere making the processed image blurry and not containing useful information on vegetation and open water covered surfaces.

In an NRG false colored image vegetation depending on types and conditions, appears in different shades of red. Due to higher green band reflectance clear water appears dark-bluish while turbid water appears cyan as a result of higher red reflectance caused by sediments. Depending on their composition bare soil, roads and buildings appear in various shades of grey, blue or yellow (Fig. 5).[3]

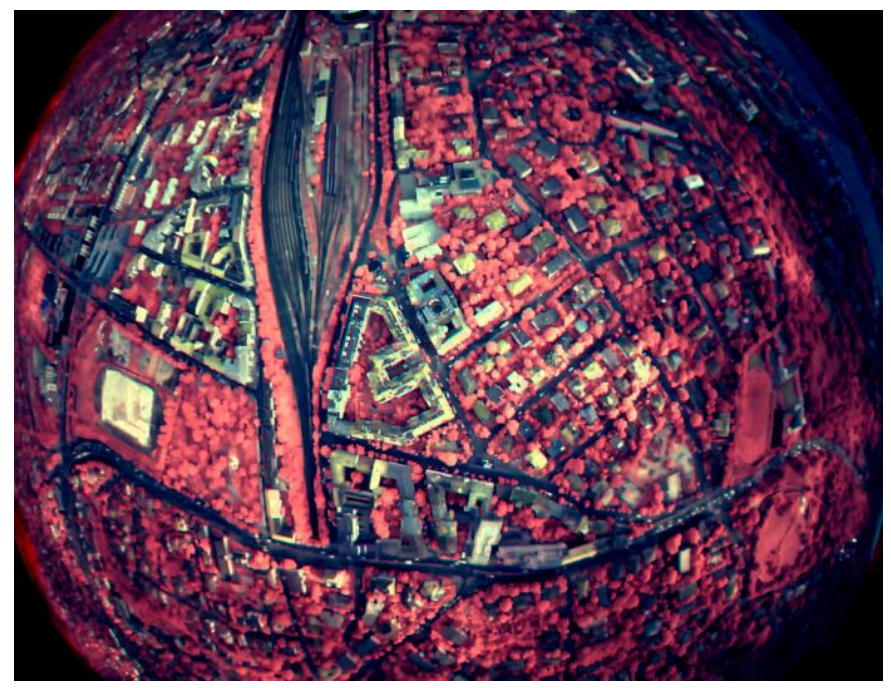

Fig. 5. NRG false color composite image of Déli Pályaudvar railway station

\section{B. NDVI Heatmap}

To measure vegetation health and density the Normalized Difference Vegetation Index (NDVI) is used which is calculated by equation (1). NDVI is varies between -1.0 and +1.0 where the more healthy, dense vegetation is indicated by a more positive value. Open water and snow covered surfaces are represented by values close to -1.0 since very little of no NIR light reflected by these areas.

NDVI is an index which measures the vegetation density and condition. It is influenced by the fractional cover of the ground by vegetation, the vegetation density and the vegetation greenness. It indicates the photosynthetic capacity of the land surface cover.

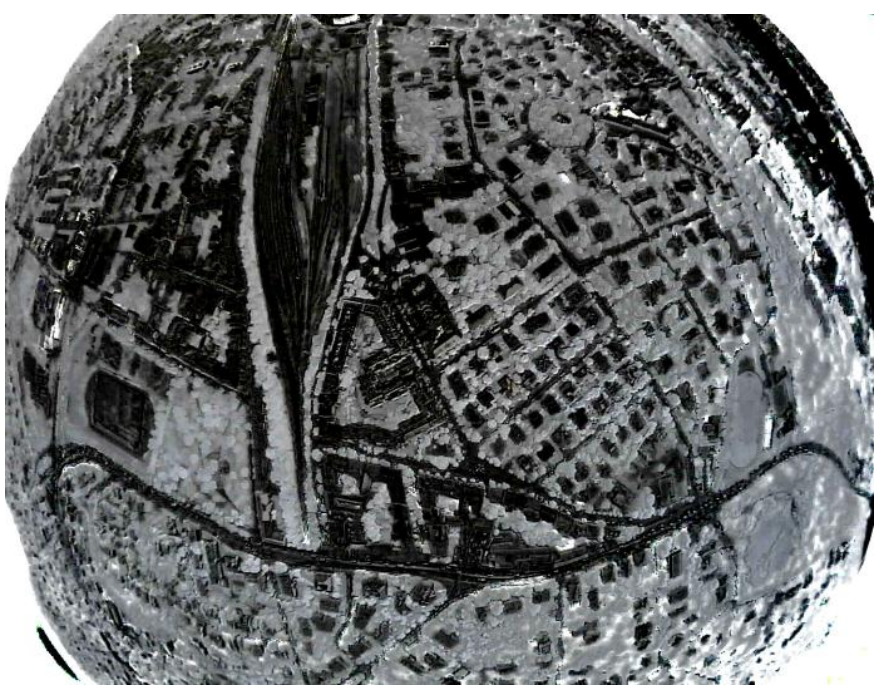

Fig. 6. NDVI heat map of Déli Pályaudvar railway station

NDVI computation is a pixel wise transformation. The generated data can be visualized as a heat map (Fig. 6), where light areas show vegetated areas and darker shades indicates non vegetated surfaces.

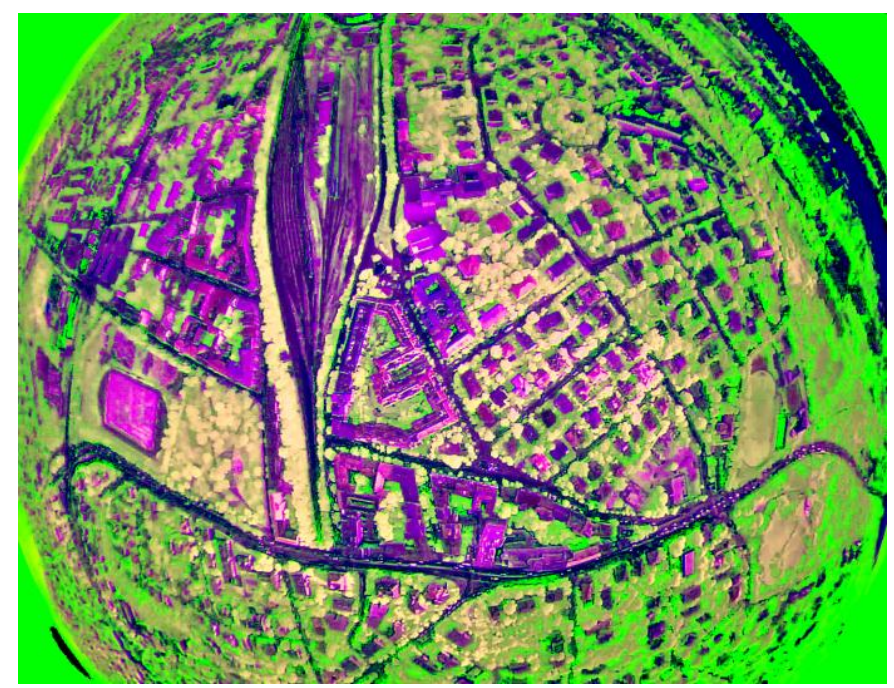

Fig. 7. NDVI Color Composite image of Déli Pályaudvar railway station

NDVI information combined with other bands captured by a multispectral camera will form a color composite image that helps differentiate vegetation types. In Fig. 7 NIR band is assigned to the Red (R) channel, NDVI to the Green $(G)$ and green band (of VIS) is assigned to the Blue channel. In this type of composite at least three types of vegetation can be discriminated: Green areas with dense, closed canopy trees, 
bright yellow with bushes or less dense trees and golden yellow areas covered with grass. Dark blue and magenta areas indicate non vegetated surfaces.[3]

\section{TEST FLIGHTS}

This year four test flights were performed with the multispectral camera prototype. The first two flights were shakedown tests with only VIS imaging. While the third and fourth were full featured flights. These flight events were originally planned in the Technological Demonstration Missions (TDM) which is a programme restricted to UPRA hardware and the goal is to provide flight time and testing opportunity for in-house developed components. TDM payload could also be connected to Fly With Us (FWU) payload train if the third party payload makes it available and not forbidden by the payload developers.

\section{A. VIS Only shakedown test}

Two flights were performed in March and April as part of the Technological Demonstration Missions (TDM) programme. The goal of TDM-1 and TDM-2 missions was to test the camera electronics and insulation in real flight condition. During the test flights both sensor modules were equipped with stock lens which reduced the performance of the payload to VIS only operation.

Both tests were successful; the payload was recovered and was operational after flight.

TDM-1 was a mid-altitude flight with the peak altitude of 14000 meters and 2 hours of flight time. Image data gathered was in focus on both sensors and due to the sunny weather conditions they were also clear with minimal or no clouds appearing in the pictures.

TDM-2 was a long duration, high altitude mission with a peak altitude of 33000 meters and 5 hours of flight time. Images taken were high quality, but due to the cloudy weather pictures taken at higher altitude were only showed small portions of landscape.

\section{B. NIR-VIS Wide angle}

After the successful shakedown tests the stock lenses were replaced with wide angle infrared capable optics. A 850nm longpass filter was added to lens of the NIR sensor while the lens of the VIS sensor left bare. The replacement procedure was performed in a 'grey' lab area to provide minimal contamination on the sensors.

Two test flights were conducted in June and early August with the full featured camera. Both flights were successful, the payload train was recovered and on-board data was retrieved.

TDM-3 was a mid-altitude flight with the peak altitude of 16000 meters and 2 hours of flight time. Images taken during the flight showed that further focus calibration would be needed on both sensors as the images appear less sharp than expected although manual image processing methods presented acceptable composite pictures of the surface.
The flight in August was a part of the Fly With Us (FWU) programme to provide flight opportunity for third party payloads. FWU-2 was a multiple payload flight with the peak altitude of 31400 meters and 2 hours of flight time.

Processing of FWU-2 flight data is still in progress in the time of writing this paper, but preliminary results show the remote sensing payload performed well. As in Fig. 8 a). can be seen the VIS image is desaturated and low on contrast which is the result of the missing $800 \mathrm{~nm}$ shortpass (infra cut) filter. This effect can be corrected during composite image generation. Manual image processing supplemented with automated overlapping shows promising results in NRG false coloring and NDVI composite generating.

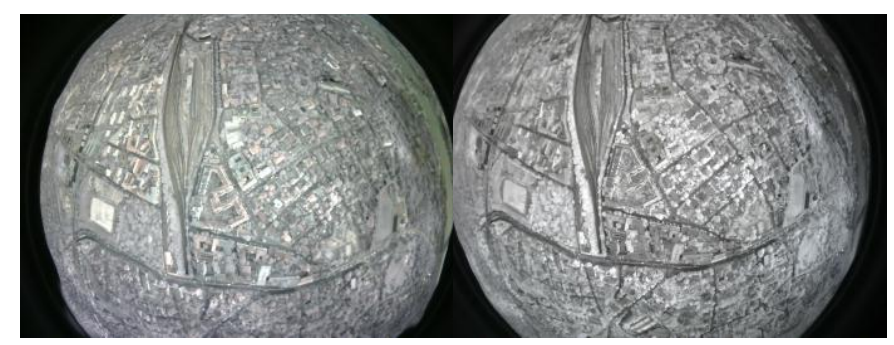

Fig. 8. a) VIS image (left), b) NIR image (right)

\section{SUMMARY}

With a stratospheric balloon remote sensing activities could be performed cost effectively in a frequent manner. Each balloon flight could cover large areas with the possibility of pin point observation. Currently UPRA Team is able to keep a two week return time if no changes required on the payload configuration which could be lowered using multiple, identical payload-trains. This could be applied for short term remote sensing campaigns that targeted to collect frequent data on a designated area.

The UPRACAM proof of concept remote sensing payload shows that developing an affordable small sized multispectral camera is possible and could be the backbone of vegetation and water observation missions. The data collected could help in crop management and agricultural planning while also could be an aid to authorities during floods, after hail or storms to organize protection or salvage.

Keeping in mind the safety, scalability and modularity of the balloon platform, the information and experience gathered during the test flights provides us guidance for adding features and functions to the UPRACAM remote sensing payload and improve the reliability of the platform..

\section{REFERENCES}

[1] Zs. Bodó, B.Góczán, Developing Balloon-Borne Payload for Remote Sensing Applications, H-Space Conference 2019

[2] OMNIVISION, "OV2640 Datasheet," OmniVision Technologies, Inc., 1341 Orleans Drive Sunnyvale, CA USA, 2006.

[3] Dr. Liew Soo Chin, Interpreting Optical Remote Sensing Images, https://crisp.nus.edu.sg/ research/tutorial/opt int.htm, 2001 